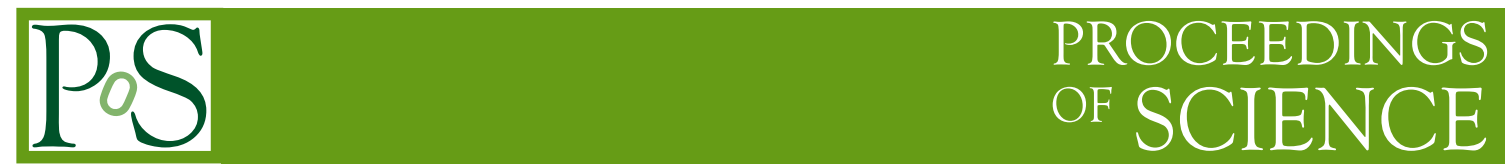

\title{
Getting there: from AMANDA to IceCube
}

\author{
Carlos P. de los Heros* \\ (for the IceCube collaboration) \\ Uppsala University, Sweden \\ E-mail: cphetsl.uu.se
}

\begin{abstract}
AMANDA is a neutrino telescope consisting of 677 optical modules embedded at $1.5-2 \mathrm{~km}$ depth in the ice cap under the Amundsen-Scott South Pole station. In this contribution we summarize AMANDA results on measuring the atmospheric neutrino spectrum, on searches for a neutrino flux from the galactic plane, for a diffuse and point-like flux of cosmic high energy neutrinos and searches for dark matter candidates in the form of MSSM neutralinos. 90\%CL limits are presented for these searches, as well as for a list of specific point source candidates.
\end{abstract}

The status and physics capabilities of IceCube, the successor of AMANDA, are also summarized.

International Europhysics Conference on High Energy Physics

July 21st - 27th 2005

Lisboa, Portugal

* Speaker. 


\section{Introduction}

On January 2005, the first string of the IceCube neutrino telescope was deployed to $2.5 \mathrm{~km}$ depth a few hundred meters from the geographical South Pole. This event marks the beginning of the construction of the first $\mathrm{Km}^{3}$ neutrino telescope in the world. IceCube builds on the expertise and technology developed for the AMANDA neutrino telescope, in operation at the South Pole since 1997. The completed detector, AMANDA-II, consists of 677 optical modules (OM), photomultiplier tubes housed in glass spheres, and deployed in 19 strings at depths between $1450 \mathrm{~m}$ and $2000 \mathrm{~m}$ The strings are arranged approximately in three concentric circles of $40 \mathrm{~m}, 100 \mathrm{~m}$ and 200 $\mathrm{m}$ in diameter. The detector is taking data in such configuration since 2000. Due to the constraints of the site, the detector was built in stages, and the first data used for physics analysis were taken between 1997 and 1999, with 302 OMs in 10 strings (AMANDA-B10).

Neutrino detection is achieved through the detection of the Cerenkov emission of secondaries produced in neutrino interactions in or around the detector. Two channels are used. Muon-neutrinos are detected by the muon produced in the interactions. Nanosecod timing resolution in the OMs allows to reconstruct the muon trajectory with a resolution of $1.5^{\circ}$ to $2.5^{\circ}$, depending on the requirements of the analysis performed. In the case of electron and tau neutrinos, the electromagnetic or hadronic cascade at the interaction vertex is detected, with worse pointing resolution of $\sim 30^{\circ}$.

The case for large-scale neutrino telescopes has been made elsewhere (see for example [1]). In this paper we will concentrate on the results obtained with AMANDA-II so far. In section 3 we give an overview of the characteristics and physics potential of IceCube.

\section{Results from AMANDA}

\subsection{Atmospheric muons}

Cosmic rays impinging on the atmosphere produce a continuous flux of muons from the decays of the produced pions and kaons. A measurement of muon intensity versus depth can be done by measuring the muon flux as a function of declination angle and correcting for the slant depth to the detector, as shown in figure 1. Note that only muons with an energy above $400 \mathrm{GeV}$ at the surface can penetrate the $1500 \mathrm{~m}$ of ice to trigger the detector. The AMANDA results [2] are compatible with previous measurements of other collaborations and compatible with a primary energy spectrum proportional to $\mathrm{E}^{-2.7}$.

The existence at the South Pole of a surface scintillator array, SPASE [3], makes it possible to do primary composition studies. SPASE measures the electron component of cosmic ray showers at the surface, while AMANDA can measure the muon component at $1500 \mathrm{~m}$ depth. These measurements can be related to the energy and mass of the primary. Results from data taken with both detectors running in coincidence [ $₫$ ] show a trend of increasing primary mass with energy, compatible with a composition change to heavier primaries around the knee $\left(\sim 10^{4} \mathrm{TeV}\right)$.

\subsection{The atmospheric neutrino flux}

The atmosphere is also a guaranteed source of neutrinos from the decay of muons, pions and kaons. AMANDA has measured the atmospheric muon neutrino flux to an energy of about 300 $\mathrm{TeV}$ as shown in figure 2. An advantage of the extended energy range covered by AMANDA is 


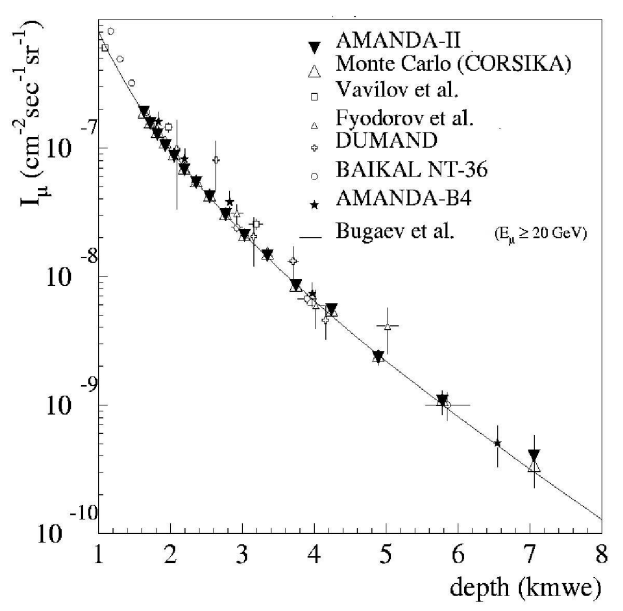

Figure 1: Atmospheric muon intensity as a function of vertical depth as measured by AMANDA. Results from other experiments are shown for comparison

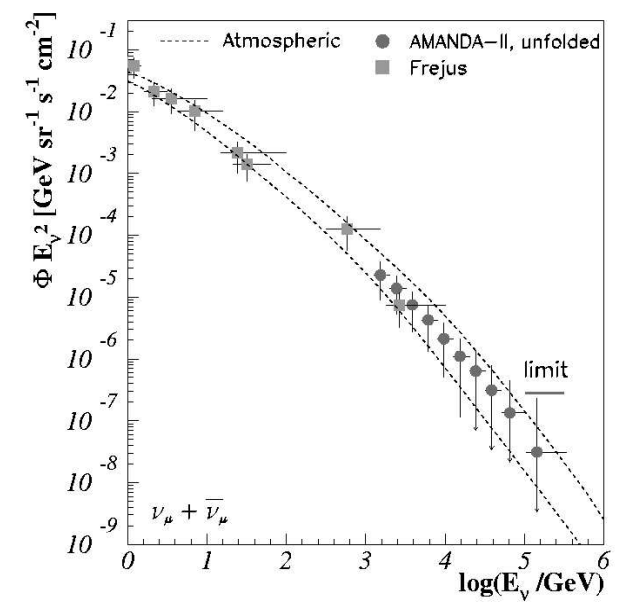

Figure 2: Atmospheric muon-neutrino energy spectrum measured by AMANDA. The results of Frejus at lower energies are shown as comparison.

that the measurement at the highest energy bin can be used to set a limit on a generic $\mathrm{E}^{-2}$ diffuse cosmic flux, since some models predict the onset of such flux at the TeV level. Using the measured atmospheric neutrino flux between 100 and $300 \mathrm{TeV}$ (last bin in figure a 90\% CL limit on a $\mathrm{E}^{-2}$ $v_{\mu}$ flux can be set at a level of $\mathrm{E}^{-2} \Phi_{v_{\mu}}<2.6 \cdot 10^{-7} \mathrm{GeV} \mathrm{cm}^{-2} \mathrm{sr}^{-1} \mathrm{~s}^{-1}$ [阿].

\subsection{The Galactic plane}

Cosmic rays interacting with the interstellar medium are another source of guaranteed neutrinos, which are produced through a similar process as with the interactions on the atmosphere: pion and kaon production and subsequent decays of these particles. However the geographical situation of AMANDA is not optimal for this kind of study. The galactic center and a good part of the galactic disk are not seen from the AMANDA site, which views only the outer region of the galactic plane, between longitude values $33^{\circ}<l<213^{\circ}$. We have used the data sample collected between 2000 and 2003, 3329 events, to search for a possible enhancement of neutrinos from the visible part of the galactic disk from the AMANDA site. Assuming a Gaussian shape of the distribution of matter in the galactic disk, with a width of $2.1^{\circ}$, and a $\mathrm{E}^{-2.7}$ spectrum, we obtain a $90 \%$ CL limit on the neutrino flux of $\Phi / \mathrm{dE}=4.8 \cdot 10^{-4} \mathrm{GeV}^{-1} \mathrm{~cm}^{-2} \mathrm{sr}^{-1} \mathrm{~s}^{-1}$ in the range $0.2 \mathrm{TeV}<\mathrm{E}_{v}<40 \mathrm{TeV}$.

\subsection{Searches for a cosmic neutrino flux}

The search for neutrinos of cosmic origin is the main physics endeavour of neutrino telescopes. There is general consensus that the most violent objects detected with conventional astronomy (active galactic nuclei, GRBs, quasars... etc.) should also be neutrino emitters. The idea is that protons accelerated in these objects will interact with the ambient matter or radiation field and produce pions. Neutral pions would produce the observed $\gamma$-rays, while the decay of charged pions would yield a neutrino flux. The discovery of a neutrino signal from such cosmic objects would provide an unambiguous confirmation of this scenario, pointing at these objects as the accelerator 


\begin{tabular}{|c|c|c|c|c|c|}
\hline data year & $\begin{array}{l}\text { lifetime } \\
\text { (d) }\end{array}$ & channel & $v$ energy range & $\begin{array}{c}\mathrm{E}^{2} \mathrm{~d} \Phi / \mathrm{dE} \text { limit } \\
\left(\mathrm{GeV} \mathrm{cm}^{-2} \mathrm{sr}^{-1} \mathrm{~s}^{-1}\right)\end{array}$ & ref. \\
\hline 1997 & 131 & muon & $6 \mathrm{TeV}-1 \mathrm{PeV}$ & $2.5 \cdot 10^{-6}$ & [6] \\
\hline 1997 & 131 & muon & $1 \mathrm{PeV}-3 \mathrm{EeV}$ & $9.9 \cdot 10^{-7}$ & [南] \\
\hline 1997 & 131 & cascade & $50 \mathrm{TeV}-3 \mathrm{PeV}$ & $9.8 \cdot 10^{-6}$ & {$[8]$} \\
\hline 2000 & 174 & cascade & $50 \mathrm{TeV}-5 \mathrm{PeV}$ & $8.6 \cdot 10^{-7}$ & {$[9]$} \\
\hline $2000-03$ & 807 & muon & $13 \mathrm{TeV}-3.2 \mathrm{PeV}$ & $2.8 \cdot 10^{-7(b, *)}$ & [10] \\
\hline 2000 & 174 & muon & $100 \mathrm{TeV}-300 \mathrm{TeV}$ & $7.8 \cdot 10^{-7(*)}$ & [5] \\
\hline
\end{tabular}

Table 1: $90 \%$ CL limits on a diffuse all-flavour cosmic neutrino flux from different AMANDA analyses. ${ }^{(b)}$ denotes sensitivity. ${ }^{*}$ denotes preliminary results. Limits 1,5 and 6 have been multiplied by three with respect to the value given in the reference in order to scale them to all-flavour limits.

sites of the highest-energy cosmic rays. Note that the proposed mechanism would produce a fhvour ratio at the source of $v_{e}: v_{\mu}: v_{\tau}=1: 1: 0$. The detection of a $v_{\tau}$ component in the cosmic neutrino flux would be a clear $v_{\tau}$ appearance observation.

Diffuse flux: Even if the neutrino flux from individual sources would turn out to be too weak to be unambiguously detected with a detector like AMANDA, a diffuse flux of neutrinos from the injected spectrum of all sources in the Universe could be detectable. The search for such flux is a challenge since it is, by definition, not correlated in time or position in the sky with any particular object. The search is based on the expected harder neutrino spectrum, $\mathrm{d} \Phi / \mathrm{dE} \propto \mathrm{E}^{-2}$, from the shockacceleration of protons in the source, as compared to the $\mathrm{E}^{-3.7}$ dependence of the atmospheric neutrino flux. This search can be done both in the muon and cascade channels, and can cover a wide range of energies, from a few tens of $\mathrm{TeV}$ to $\mathrm{EeV}$. Above PeV energies, the Earth becomes opaque to neutrinos and the search has to be concentrated on events from near or above the horizon.

A first search for a diffuse cosmic neutrino flux was performed with AMANDA-B10, corresponding to $131 \mathrm{~d}$ of lifetime from the 1997 data set, while the latest limit obtained by AMANDA is based on the analysis of four years of data, with a total lifetime of 807 days. The results of these analyses, as well as other analyses performed using the cascade channel, are summarized in table 2.4. Results marked with an asterisk should be taken as preliminary. The results of these searches are also summarized in figure 3, where they are compared to results from Baikal [11] and several theoretical predictions: the Waxman-Bahcall limit [12] and to two models of neutrino production in AGN and GRBs, the Stecker-Salamon (SS) model [13] and the Mannheim-ProtheroeRachen (MPR) model [14].

Point sources: The search for point sources of neutrinos in the sky is performed by looking for statistical excesses of events in narrow angular regions in the sky, determined by the angular resolution of the detector (about $2^{\circ}$ for this analysis). The search can be done in a generic way, looking for 'hot spots' with respect to the average background, or by looking into the position of candidate objects. In the latter case, the background is estimated from the data off-source, in the same declination band of the candidate object. For this search we have used sources to be known to be 


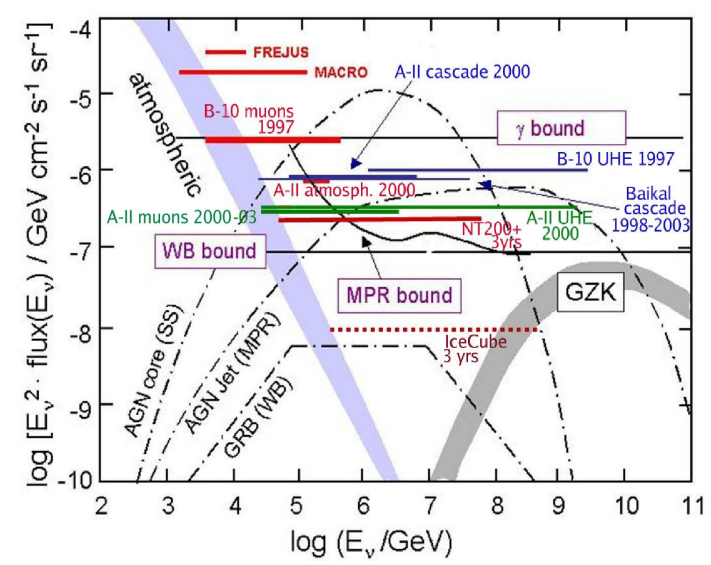

Figure 3: $90 \% \mathrm{CL}$ all-flavour flux limits as a function of energy obtained with different AMANDA analyses, compared to some theoretical predictions (the A-II line for 2000-2003 denotes sensitivity). The sensitivity of 3 years of IceCube exposure is also indicated.

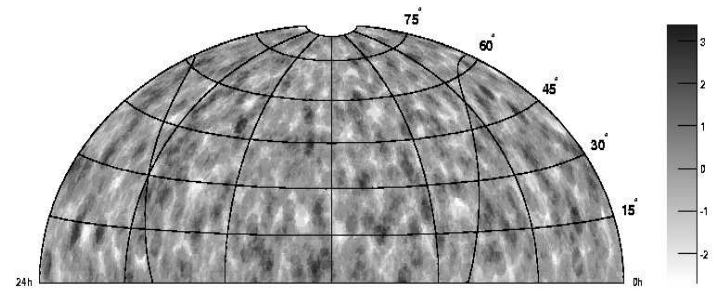

Figure 4: Significance sky map using 4 years of AMANDA data. The figure shows the deviation from a uniform background, the scale comprising $-3 \sigma$ (white) to $+3 \sigma$ (black).

gamma and/or X-ray emmitters, like Blazars, microquasars or supernova remnants. These searches are done exclusively in the muon channel due to its better pointing resolution, and therefore are restricted to objects in the northern sky.

The results summarized in this paper were obtained with the combined data sets of the years 2000-2003. It amounts to a total of $807 \mathrm{~d}$ of livetime, and the sample contains 3369 neutrino events, while the expectation from atmospheric neutrinos is 3438 events. Figure 4 shows the significance map of the northern sky in galactic coordinates. The map is compatible with a random distribution of sources, the hottest spot having a $92 \%$ probability of being a random fluctuation. Table 4.4 shows the neutrino flux limits obtained for a few selected sources assuming an $\mathrm{E}^{-2}$ neutrino energy spectrum. A total of 33 objects have been checked (see [15] for details of this analysis).

The sensitivity for point sources can be increased by using a source-stacking analysis. In this kind of analysis the data from the directions of sources showing similar emission and morphological characteristics (ie. sources from which one expects a similar neutrino production scenario and flux level with respect to their photon emission) are added. The background for each object is estimated off-source from the same zenith band as the location of the object, and added for all candidates. A preliminary analysis performed with data collected in 2000-2003 for several types of sources shows no excess over the expected Poisson statistics [16]. However the sensitivity has been improved by a factor of three for a generic AGN with respect to the point source analysis mentioned earlier in this section.

Gamma-ray bursts: A rather special type of candidate neutrino point source are Gamma Ray Bursts (GRB), since for these objects one has the time stamp of the occurrence of the event. This 


\begin{tabular}{|c|c|c|c|c|c|c|c|c|c|c|c|}
\hline Candidate & $\delta\left(^{\circ}\right)$ & $\alpha(\mathrm{h})$ & $n_{\text {obs }}$ & $n_{b}$ & $\Phi_{v}^{\lim }$ & Candidate & $\delta\left(^{\circ}\right)$ & $\alpha(\mathrm{h})$ & $n_{\mathrm{obs}}$ & $n_{b}$ & $\Phi_{v}^{\lim }$ \\
\hline \multicolumn{6}{|c|}{ TeV Blazars } & \multicolumn{6}{|c|}{ GeV Blazars } \\
\hline Mark & 38.2 & 11.07 & 6 & 5.6 & 0.68 & $19+428$ & 42.9 & 2.38 & 4 & 4.3 & 0.54 \\
\hline Mark & 39.8 & 16.90 & 5 & 5.0 & 0.61 & QSO 0954+556 & 55.0 & 9.87 & 2 & 5.2 & 0.22 \\
\hline \multicolumn{6}{|c|}{ Microquasars } & \multicolumn{6}{|c|}{ SNR \& Pulsars } \\
\hline SS433 & 5.0 & 19.20 & 2 & 4.5 & 0.21 & SGR $1900+14$ & 9.3 & 19.12 & 3 & 4.3 & 0.35 \\
\hline Cygnus X3 & 41.0 & 20.54 & 6 & 5.0 & 0.77 & Crab Nebula & 22.0 & 5.58 & 10 & 5.4 & 1.3 \\
\hline GRS $1915+105$ & 10.9 & 19.25 & 6 & 4.8 & 0.71 & Geminga & 17.9 & 6.57 & 3 & 5.2 & 0.29 \\
\hline Cygnus X1 & 35.2 & 19.97 & 4 & 5.2 & 0.40 & Cassiopeia A & 58.8 & 23.39 & 4 & 4.6 & 0.57 \\
\hline
\end{tabular}

Table 2: Results from the search for neutrinos from selected objects. $\delta$ is the declination in degrees, $\alpha$ the right ascension in hours, $n_{o b s}$ is the number of observed events and $n_{b}$ the expected background. $\Phi_{v}^{\lim }$ is the $90 \% \mathrm{CL}$ upper limits in units of $10^{-8} \mathrm{~cm}^{-2} \mathrm{~s}^{-1}$ for a spectral index of 2 and integrated above $10 \mathrm{GeV}$. These results are preliminary (the systematic errors are under assessment).

allows to perform a practically background-free analysis using both off-source and off-time background estimation. An additional advantage of having the time stamp of the event is that the required pointing resolution can be relaxed and then the cascade channel can be used, having then access to full sky searches. We have used the BATSE and IPN3 burst catalogues and looked for neutrino bursts within a few seconds of the gamma burst using the Waxman-Bahcall model as a benchmark. We have also looked for a precursor signal, up to about $100 \mathrm{~s}$ before the burst, as proposed by other models [17]. The results from the analysis of 139 bursts using the muon channel and data from 2000-2003 yield a flux limit of $\mathrm{E}^{2} \Phi_{v} / \mathrm{dE}=3 \times 10^{-8} \mathrm{GeV} \mathrm{cm}^{-2} \mathrm{~s}^{-1} \mathrm{sr}^{-1}$, while a preliminary analysis using the cascade channel on 73 bursts on 2000 yields $\mathrm{E}^{2} \Phi_{v} / \mathrm{dE}=9.5 \times 10^{-7}$ $\mathrm{GeV} \mathrm{cm}^{-2} \mathrm{~s}^{-1} \mathrm{sr}^{-1}$. The search for precursor bursts, using 50 burst from 2001-2003, yields a limit of $\mathrm{E}^{2} \Phi_{v} / \mathrm{dE}=5 \times 10^{-8} \mathrm{GeV} \mathrm{cm}^{-2} \mathrm{~s}^{-1} \mathrm{sr}^{-1}$.

\subsection{Search for dark matter candidates}

If the dark matter halo of our galaxy is composed of weakly-interacting relic stable particles, they can accumulate gravitationally in the center of heavy objects, like the Sun or Earth. The annhilitations of such particles can yield an excess of neutrinos from these objects, detectable with neutrino telescopes. The neutralino, a stable particle predicted in the Minimal Supersymmetric Standard Model, is a good candidate for dark matter. We have performed a search for neutralinos from the center of the Earth [18] and the Sun [19]. The non-observation of an excess flux has been translated into a muon flux limit. The results are shown in figures $\$$ (search from the Earth) and 6 (search from the Sun). The crosses are predictions from the MSSM, using the DarkSusy package [20]. The dots are models disfavoured by direct searches [21].

\section{Status and potential of IceCube}

IceCube is the $\mathrm{km}^{3}$ extension of AMANDA, consisting of 80 strings and 4800 optical modules arranged in an hexagonal pattern of $1 \mathrm{~km} \times 1 \mathrm{~km} \times 1 \mathrm{~km}$. The main difference with respect to AMANDA is the use of digital technology in the ice: the photomultiplier signals are digitized insitu at the optical module, which has full waveform recording capabilities with a dynamic range 


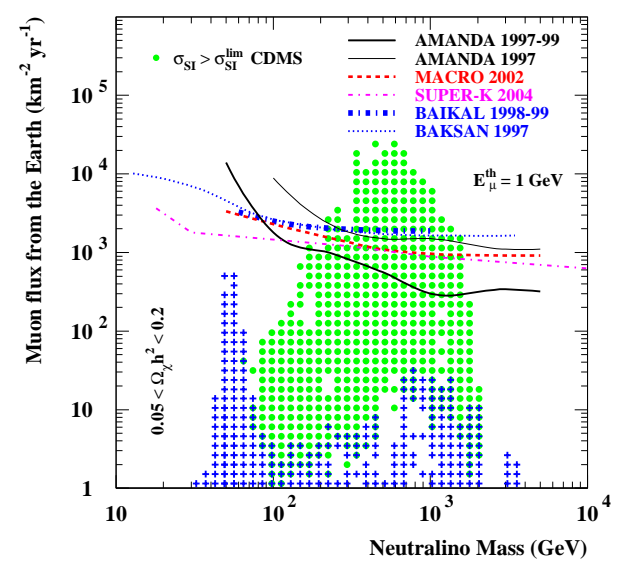

Figure 5: 90\% CL muon flux limit from neutralino annihilations in the center of the Earth, as a function of neutralino mass. Results from other experiments are shown for comparison.

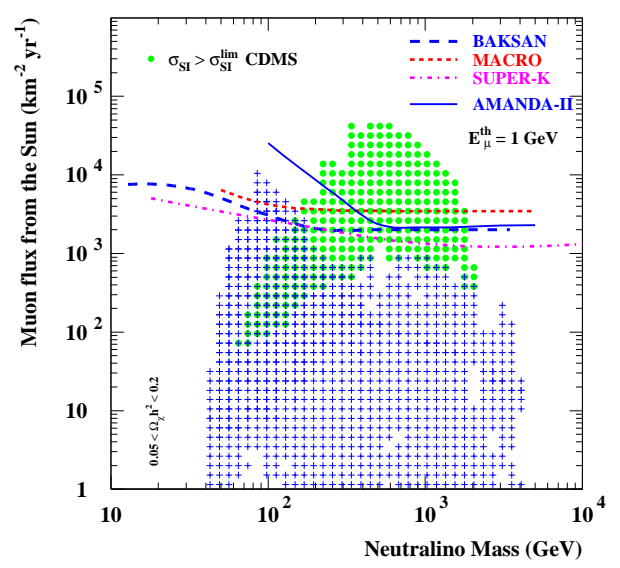

Figure 6: $90 \%$ CL muon flux limit from neutralino annihilations in the Sun, as a function of neutralino mass. Results from other experiments are shown for comparison.

between 200 and 2000 photoelectrons depending on the integration time. IceCube is expected to improve the sensitivity of AMANDA by a few orders of magnitude [22]. After 3 years of operation an all-fhvour sensitivity at the TeV-PeV energy range of $O 10^{-9} \mathrm{GeV} \mathrm{cm}^{-2} \mathrm{~s}^{-1}$ can be achieved (see figure B). The angular resolution will be considerably improved as well, reaching below $1^{\circ}$. Figures 7 and 8 show the IceCube effective area and the pointing resolution for muon-neutrino induced events for different energy ranges, as a function of zenith angle.

The IceCube project includes a surface array, IceTop, consisting of 80 stations, one at each IceCube string. A station consists of two ice tanks with two OMs each, which will detect charged particles from cosmic showers. This will extend the physics capabilities of IceCube and will also be used for calibration. The first four stations of IceTop are in place and, as of January 2005, the first string of IceCube is deployed. Construction will continue with the deployment of up to 16 strings per year, until the year 2010. But the detector will be useful for physics from next season, when the number of modules of IceCube will be comparable to the present AMANDA array.

Acknowledgments: The U.S. National Science Foundation, the University of Wisconsin Alumni Research Foundation, the U.S. DoE, the U.S. NERS Computing Center, the UCI AENEAS Supercomputer Facility, the Swedish Research Council, the Swedish Polar Research Secretariat, the Knut and Alice Wallenberg Foundation (Sweden), the German Federal Ministry of Education and Research, the Deutsche Forschungsgemeinschaft, the IWT (Belgium), the FWO(Belgium), the FNRS (Belgium) and the OSTC (Belgium). D. F. Cowen acknowledges the support of the NSF CAREER program. M. Ribordy acknowledges the support of the SNF (Switzerland).

\section{References}

[1] F. Halzen. Nucl. Phys. Proc. Suppl. B145, 301, 2005. 


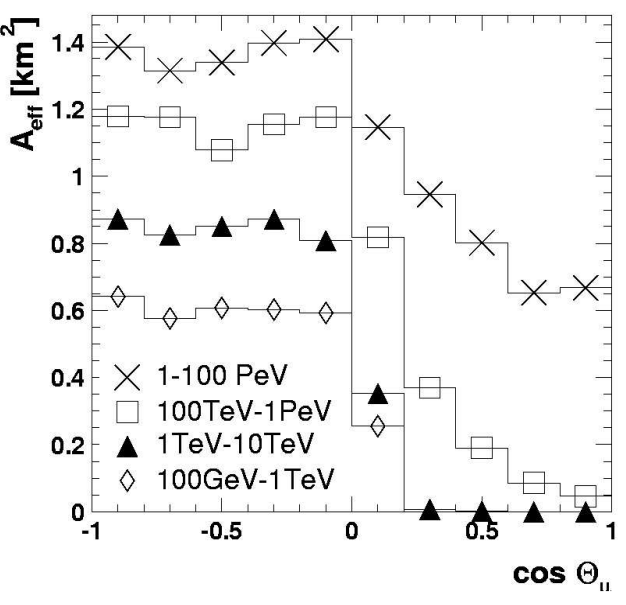

Figure 7: Effective area of IceCube for different energy regimes, as a function of cosine of the zenith angle.

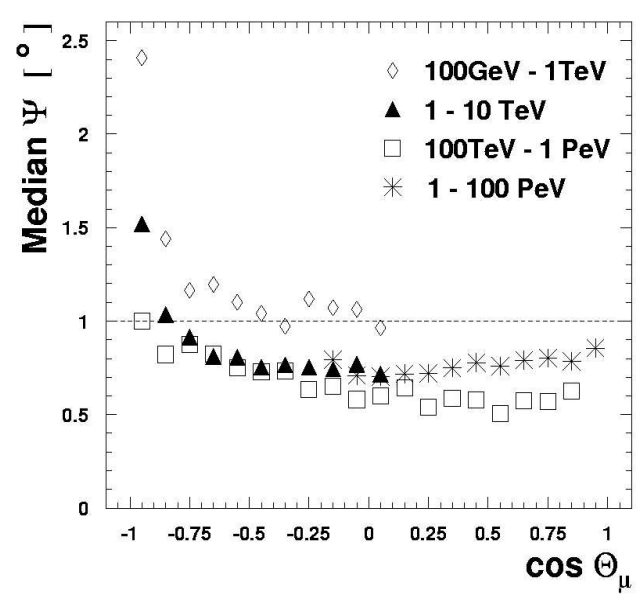

Figure 8: Pointing resolution of IceCube for different energy regimes, as a function of cosine of the zenith angle.

[2] P. Desiati et al. in proceedings of 28th Intl. Cosmic Ray Conference, 31 July - 7 August 2003, Tsukuba, Japan, HE 2.3, 1373-1376.

[3] J. E. Dickinson et al. Nucl. Instrum. Meth. A440, 95-113, 2000.

[4] J. Ahrens et al. Astropart. Phys. 20 (2004) 565-581

[5] K. Muenich et al. in proceedings of 29th Intl. Cosmic Ray Conference, 3-10 August 2005, Pune, India.

[6] J. Ahrens et al. Phys. Rev. Lett. 90, 251101, 2003.

[7] M. Ackermann et al. Astropart. Phys. 22, 339, 2005.

[8] J. Ahrens et al. Phys. Rev. D67, 012003, 2003.

[9] M. Ackermann et al. Astropart. Phys. 22, 127, 2004.

[10] J. Hodges et al. in proceedings of 29th Intl. Cosmic Ray Conference, 3-10 August 2005, Pune, India.

[11] V. Aynutdinov et al. in proceedings of 29th Intl. Cosmic Ray Conference, 3-10 August 2005, Pune, India. [astro-ph/0507712]. See also [astro-ph/0508675]

[12] E. Waxman and J. Bahcall. Phys. Rev. D59, 023002, 1999, and Phys. Rev. D64, 023002, 2001.

[13] F. W. Stecker and M. H. Salamon. Space. Sci. Rev. 75, 341, 1996.

[14] K. Mannheim, R. J. Protheroe and J. P. Rachen. Phys. Rev. D63, 023003, 2001.

[15] M. Ackermann et al. Phys. Rev. D71, 077102, 2005; M. Ackermann et al. in proceedings of 29th Intl. Cosmic Ray Conference, 3-10 August 2005, Pune, India.

[16] A. Gross et al. in proceedings of 29th Intl. Cosmic Ray Conference, 3-10 August 2005, Pune, India.

[17] S. Razzaque, P. Meszaros and E. Waxman. Phys. Rev. D68, 083001, 2003.

[18] P. Ekström. PhD thesis, Stockholm University, 2004. ISBN 91-7265-886-X; D. Hubert et al. in proceedings of 29th Intl. Cosmic Ray Conference, 3-10 August 2005, Pune, India.

[19] M. Ackermann et al. Accepted for publication in Astropart. Phys. [astro-ph/0508518]

[20] P. Gondolo et al. JCAP 0407, 008, 2004. [astro-ph/0406204]

[21] D. S. Akerib et al. Phys. Rev. Lett. 93, 211301, 2004.

[22] J. Ahrens et al. Astropart. Phys. 20, 507-532, 2004. [astro-ph/0305196] 